Journal of Engineering and Applied Sciences 14 (11): 3719-3727, 2019

ISSN: 1816-949X

(C) Medwell Journals, 2019

\title{
Efficient Energy Consumption DCT-Based Image Compression in Visual Sensor Network
}

\author{
Khamees Khalaf Hasan, Ibrahim Khalil Salih and Kamil Jadoaa Ali \\ Department of Electrical Engineering, Faculty of Engineering, University of Tikrit, Tikrit, Iraq
}

\begin{abstract}
For future wireless access technologies, the furthermost significant challenges will be the requirement to process and transmit a huge volume of image data wirelessly. One method to relieve this difficulty is by compression techniques to decrease the magnitude of transmitted image data over the wireless channel. Discrete Cosine Transform DCT utilized as a kernel transform coding of Joint Photographic Experts Group (JPEG) compression technique. DCT packing the image signal energy into few numbers of transformed coefficients associated with low frequencies. The high frequency coefficients may be discarded with little loss in image signal energy and that will not effect that much on the reconstructed image quality, since, the human eye doesn't sense the high frequency components. Using a modified version of JPEG according to the energy requirement of Visual Sensor Network VSN an adapted JPEG method is proposed. This involves the concept of processing only the low frequency portion of each block of the DCT coefficients of a given image. The compression algorithm in the proposed JPEG scheme minimizes the computational complexity and reduces the required energy consumption needed to transmit an image while it allows a trade-off between image distortion using Peak Signal to Noise Ratio PSNR metric and Compression Ratio CR. When the number of retained useful low frequency coefficient is $30 \%$ and less, the reconstructed images shows a noticeable degradation at all which can be used to counter severe hardware constraints of various wireless devices applications.
\end{abstract}

Key words: JPEG, discarding process, DCT, retained coefficients, VSN, CR

\section{INTRODUCTION}

Recently, a substantial portion of wireless data is comprised of images. Most of the VSN applications are centered towards harvesting image information from the physical environment, performing simple processing on the extracted data and transmitting it to remote locations as shown in Fig. 1 (Nasri et al., 2010).

Widespread use of mobile devices and wireless sensors require algorithms that can comply with the representation of the input data in a proper form for storage and transmission. One approach to overcome this problem is to eliminate redundant information from the

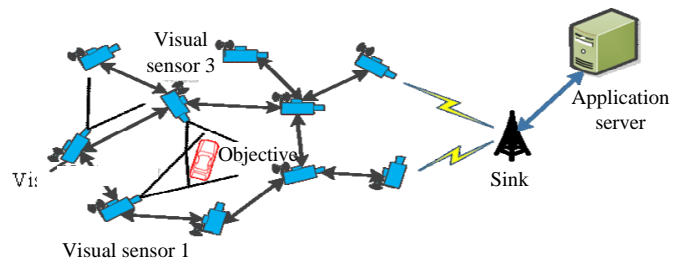

Fig. 1: Visual sensor network architecture (Nasri et al., 2010) transmitted images or frames of data over the wireless channel through image compression techniques (Nasri et al., 2010). Typically, visual information needs to be compressed using some standards such as the (JPEG) or (JPEG2000). These algorithms need very complex hardware requirements. However, these efforts fall short of meeting real-time processing requirements. Using an adapted version of JPEG according to the energy requirement of VSN, a new method is proposed. This involves the concept of processing only the upper-left portion of each block of the DCT coefficients of a given image. This is followed by modifying the parameters on the computation time and communication energy. Nevertheless, JPEG has many restrictions using DCT, especially, at portable low bit-rate applications. The energy compaction property of DCT was used in (Taylor and Dey, 2001) but they investigated this idea in mobile wireless multimedia networks requirements. Chiasserini et al. (2002) implemented JPEG with integer DCT kernel as compression algorithm. The research did not certainly familiarize JPEG to the VSN energy requirement. Pekhteryev et al. (2005) studied only the transmission of JPEG images over the applied wireless

Corresponding Author: Khamees Khalaf Hasan, Department of Electrical Engineering, Faculty of Engineering, University of Tikrit, Tikrit, Iraq 
communication standard IEEE 802.15.4 which outlines the Zigbee nodes. The idea of minimizing the processing and transmission energy when executing JPEG in VSN was explored by Mammeri et al. (2008a, b) where the DCT compaction used a slice of each block of DCT coefficients, the selected coefficients slice is triangular. Mammeri et al. (2008a, b) used the square type of the selected coefficients portion but they do not consider how the values of the coefficients are selected. Mammeri et al. (2008a, b) proposed two methods for selecting this value in the case of triangular selection. The squared approach was not investigated. Both squared and triangular type of the DCT coefficients portion to be processed by Makkaoui et al. $(2010 \mathrm{a}, \mathrm{b})$ but did not propose any method for selecting the size, (i.e., the number of DCT coefficients). Fast DCT algorithms as well as zonal techniques have been intensively studied in the literature (Loeffler et al., 1989; Feig and Winograd, 1992; Bracamonte et al., 1996; Liang and Tran, 2001; Taylor and Dey, 2001; Jeong et al., 2004; Ferrigno et al., 2005; Hsieh and Zonal, 2005) there is no work of them that combines both squared and triangular approaches, especially in the wireless networks context. In this study, the proposed algorithm for handling this constraint problem consists of adapting JPEG to the energy requirement of VSNs. This is done by processing only a portion of each block of DCT coefficients of the captured image using the effects of selecting the size (the number of low frequency DCT coefficients) of the portion to be processed on other performance metrics like the energy consumption using the discarding process as an alternative of the quantization process of high frequency components in traditional JPEG.

\section{MATERIALS AND METHODS}

Through that the image adjacent pixels are highly correlated, these unnecessary details can be rejected by finding a less correlated representation of the image using transform coding. The very stunning and public transform-based image processing and compression methods such as JPEG using DCT (Loeffler et al., 1989). A DCT encoder transforms the picture blocks and converts the signals into frequency components (Feig and Winograd, 1992). The DCT has a robust energy compaction and brilliant decorrelation property (Bracamonte et al., 1996; Liang and Tran, 2001). Most of the signal information tends to be concentrated in a few low-frequency components as shown in Fig. 2. The low frequency components remains, so that can be used in the reconstruction process (decompression).

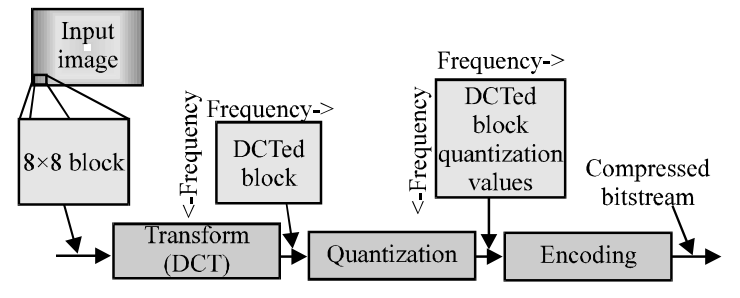

Fig. 2: Energy compaction property of DCT blocks Chew et al., 2008)

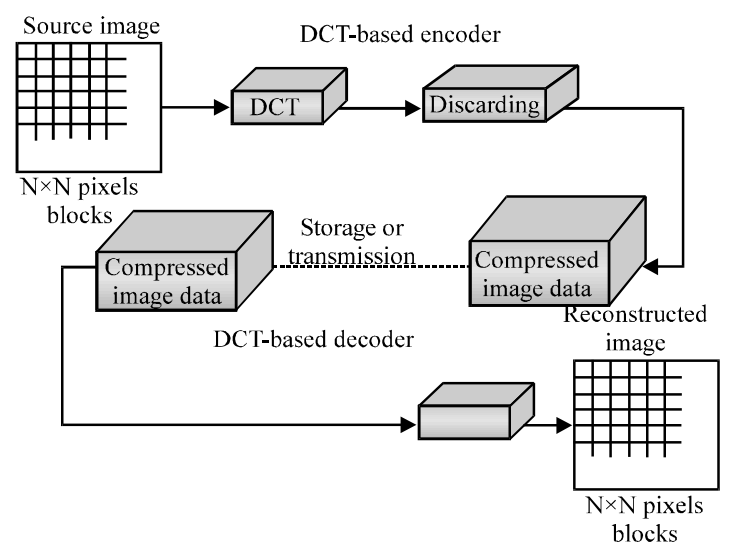

Fig. 3: Proposed DCT compression system

Since, the human eye will not notice or senses the high frequency components in the image, so, this gives the DCT an advantage of discarding the redundant data without affecting that much on the image. The less important (high) frequency components are discarded from the image in quantization process of high frequency components, hence, the use of lossy JPEG method for compression as shown in Fig. 2. Referring to JPEG system, discarding process as an alternative of the quantization process of high frequency components in the proposed adaptive JPEG method as shown in Fig. 3 which illustrate the main stages of DCT algorithm that proposed in this research. At the receiving point these discarded DCT coefficient are inverse transformed using the inverse DCT to reconstruct the image. JPEG image compression scheme based on the DCT are very popular but this transform is computationally intensive and hence is energy consuming. Unfortunately, the resource limitation of sensor nodes in VSNs in terms of memory or processor speed makes most of the compression algorithms inapplicable. The proposal presents a methodology to reduce the energy consumption of VSN node. This methodology allows a trade-off between energy consumption and image distortion. Actually, a number of applications have a preference for saving computation energy over image quality for remote VSN application 


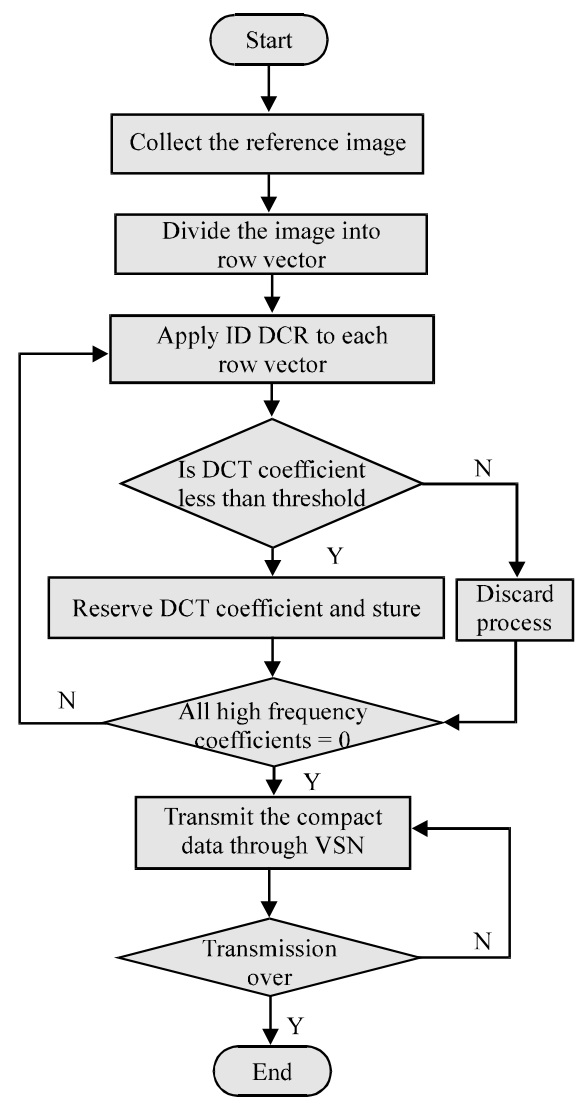

Fig. 4: Adapted JPEG image compression algorithm for the VSN

while other applications focus on high quality feature of images rather than the amount of energy consumed (Akyildiz et al., 2007; Wu and Abouzeid, 2005; Srisooksai et al., 2012; Hasan et al., 2014).

Even though the DCT process is unable to compress the input images, it is the computation-intensive principal segment of the DCT-based image compression system that promotes energy consumption. Therefore, this study focuses on the DCT transform phase in order to decrease energy consumption. Thus, decreasing the amount of transmitted data will reduce communication energy and hence, expand the general wireless network lifetime. From the block diagram shown in Fig. 4 of the suggested JPEG image compression algorithm for the VSN requirements, the image first transformed into DCT coefficients where the low frequency components kept, so, these coefficients can be used in the reconstructing process and the high components are discarded, then these coefficients are stored or transmitted in DCT coding form. The proposed DCT system used in this study uses the discarding theory instead of the quantization as shown in Fig. 4.
The proposed system consists of five main stages, firstly, the encoding process of the input captured image by dividing the original image into two fields. Then each line in the field is divided into blocks of the same length. Each block is considered as a row vector. 1D DCT is applied to transform each row vector from spatial domain to frequency domain with the same dimension to be represented in a more compact form. The few low frequency coefficients represent the DCT packing of the image energy where the high frequency coefficient can be discarded with little loss in energy, the discarded coefficients are replaced by zero coefficients.

In the proposed system, the applied test images of size $(256 \times 256)$ with 256 gray levels, each line in the images is divided into two blocks. Each block is considered as a row vector of 128 pixels. 1D DCT is applied to convert each vector of pixels into a vector of 128 transformed coefficients. 1D DCT packing the energy into few numbers of transformed coefficients associated with low frequencies, the high frequency coefficients are discarded and replaced by zero coefficients.

In this system, we separate the image using DCT firstly into two blocks of $(256 \times 128)$ dimension, then we obtain the coefficients in the same dimension from column (1-128) in the first block and from column (129-256) in the second block. The energy compaction is in the first, little columns, so, we will delete the column that contain the very high frequency coefficients and will not transmit them. These important coefficients will feed into inverse DCT system to get the advantage of the combination with the transformed coefficients. Then most of the high frequency component were deleted in a sequence of steps testing the system performance while increasing the compression ratio as trade off the distortion measure PSNR.

\section{RESULTS AND DISCUSSION}

In the proposed DCT system using discarding theory of high frequency components that has very low coefficient values in the transformed image, three test images with different contains and details were used to evaluate the systems performance: langair image, bridge image and flowers image. Two performance measurements have been employed, namely the objective and subjective. The most widely measurement used for the objective quality image processing are the Mean Square Error (MSE) and Peak Signal to Noise Ratio (PSNR) and this is measured in $\mathrm{dBs}$ :

$$
\operatorname{MSE}=\frac{1}{\mathrm{~N}} \sum_{\mathrm{i}=1}^{\mathrm{N}}\left(\mathrm{x}_{\mathrm{i}}-\mathrm{y}_{\mathrm{i}}\right)^{2}
$$




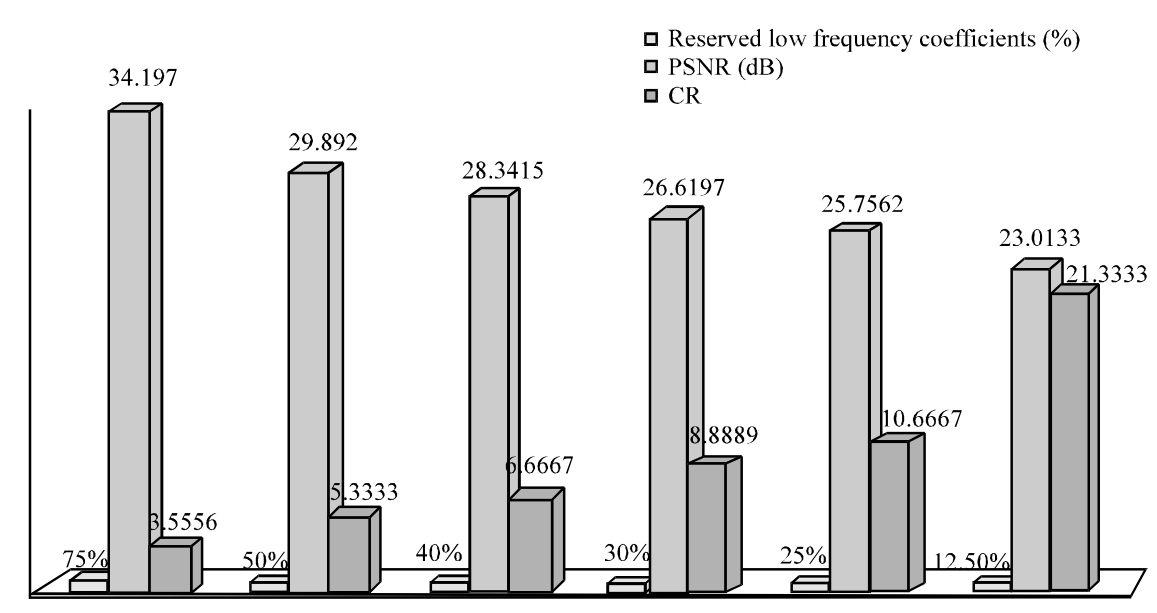

Fig. 5: CR and PSNR results of image "Langair"

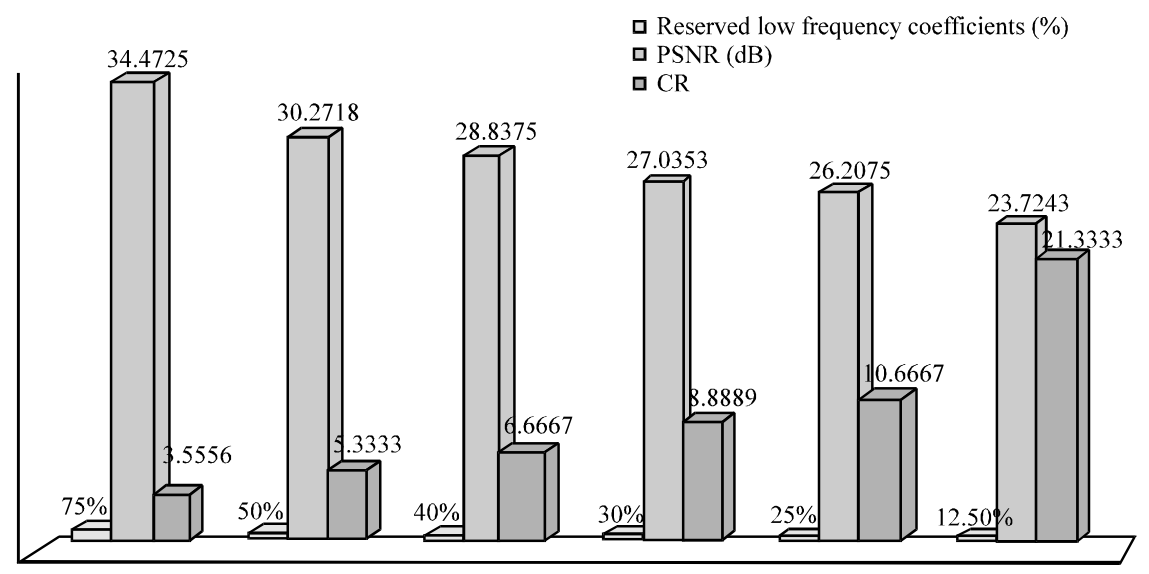

Fig. 6: CR and PSNR results of image "Bridge"

$$
\text { PSNR }=10 \log _{10} \frac{\mathrm{L}^{2}}{\mathrm{MSE}}
$$

Where:

$\mathrm{N} \quad=$ The number of pixels in the image

$\mathrm{x}_{\mathrm{i}}$ and $\mathrm{y}_{\mathrm{i}}=$ The ith pixel in the original and reconstructed image signals, respectively

L = The dynamic range of the pixel (for 8 bits per pixel grayscale image $\mathrm{L}$ is equal to 255 )

At times compression is as an alternative measured by asserting the bit rate achieved by compression in bpp (bits per pixel) and is defined by:

$$
\mathrm{BR}=\frac{\text { Original image size in bit }}{\text { No of pixels of the image }}
$$

By applying the MATLAB program on these pictures this will yields to results showing the PSNR, Mean Square
Table 1: Objective measurement of image "Langair"

\begin{tabular}{lcccc}
\hline $\begin{array}{l}\text { Reserved } \\
\text { coefficients (\%) }\end{array}$ & PSNR (dB) & MSE & CR & BR (bpp) \\
\hline 75 & 34.4725 & 23.21840 & 3.55560 & 2.2500 \\
50 & 29.8920 & 61.08010 & 5.33330 & 1.5000 \\
40 & 28.8375 & 84.98200 & 6.66670 & 1.2000 \\
30 & 27.0353 & 128.6923 & 8.88890 & 0.9000 \\
25 & 26.2075 & 155.7158 & 10.6667 & 0.7500 \\
12.5 & 23.7243 & 275.8369 & 21.3333 & 0.3750 \\
\hline
\end{tabular}

Table 2: Objective measurement of image "Bridge"

Reserved

\begin{tabular}{lcccc} 
coefficients (\%) & PSNR (dB) & MSE & CR & BR (bpp) \\
\hline 75 & 34.1970 & 24.73880 & 3.55560 & 2.2500 \\
50 & 30.2718 & 66.66160 & 5.33330 & 1.5000 \\
40 & 28.3415 & 95.26400 & 6.66670 & 1.2000 \\
30 & 26.6197 & 141.6149 & 8.88890 & 0.9000 \\
25 & 25.7562 & 172.7656 & 10.6667 & 0.7500 \\
12.5 & 23.0133 & 324.9001 & 21.3333 & 0.3750 \\
\hline
\end{tabular}

Error (MSE), CR and Bit Rate (BR). All these objective measurements plus the reconstructed image show how much the compression will effect on the image quality. Table 1-3 and Fig. 5-7 shows the values of (the objective 


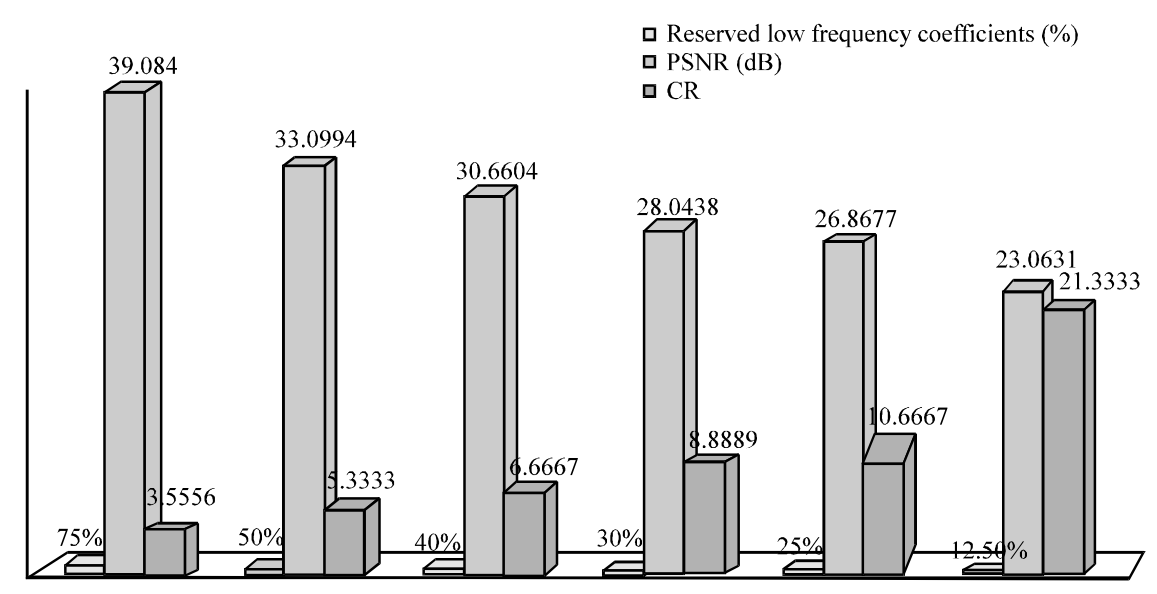

Fig. 7: CR and PSNR results of image "flower"

\begin{tabular}{|c|c|c|c|c|}
\hline $\begin{array}{l}\text { Reserved } \\
\text { coefficients (\%) }\end{array}$ & $\operatorname{PSNR}(\mathrm{dB})$ & MSE & CR & $\mathrm{BR}(\mathrm{bpp})$ \\
\hline 75 & 39.0840 & 24.7388 & 3.5556 & 2.2500 \\
\hline 50 & 33.0994 & 66.6616 & 5.3333 & 1.5000 \\
\hline 40 & 30.6604 & 95.2640 & 6.6667 & 1.2000 \\
\hline 30 & 28.0438 & 141.6149 & 8.8889 & 0.9000 \\
\hline 25 & 26.8677 & 172.7656 & 10.6667 & 0.7500 \\
\hline 12.5 & 23.0631 & 324.9001 & 21.3333 & 0.3750 \\
\hline
\end{tabular}

measurement) of each test image used in the system with different selected numbers of retained low frequency DCT coefficients as these coefficients decreases the compression ratio increases but with trade off the image quality of the reconstructed one by monitoring the PSNR decreases with noticeable degradation in image quality especially at the edges. Generally, when the bit rate decreases at low bit rate transmission of the image, the PSNR will decreases and the quality will be corrupted by smearing in the edges.

Figure 8-10b-g show the reconstructed images using $(75,50,40,30,25$ and $12.5 \%)$ low frequency coefficients, respectively. When the number of retained coefficient is 64 at bit rate $1.5 \mathrm{bpp}$, the reconstructed images shows a noticeable degradation at all. When the retained coefficient is taken to be (32 or 16) the reconstructed images show a noticeable smearing specially in the edges.

A comparison between the three reconstructed images using $(75,50,40,30,25$ and $12.5 \%)$ low frequency coefficients, respectively is shown in Fig. 11.

In order to evaluate the welfares of our proposal, we established a simplified energy consumption model for JPEG image transmission scheme. In this study, we offered an adaptive JPEG image compression model for VSNs based in DCT transmission and decomposition to achieve the energy conservation. According to the VSN constraints, this proposal is evidently relaxed to implement, allowing independent and self-adaptive performance of VSN sensor nodes and providing a compromise amongst dissipated energy and received image quality over the VSN. Image data is usually transmitted in further than one data packet. Once, captured image raw data is encoded (applying 1D DCT) and packetized into different priorities $(75,50,40,30,25$ and $12.5 \%$ ), the data packets are then ready to be sent. The source sensor transmits the data packets beginning by those with the maximum priority, then continues with those of the next minor priority and so on continuing in the same way. This selection is driven by the constraint energy in the context of VSN. The utmost important parameters are PSNR and CR. These parameters will be tested during the compression process to make the decision to choice the optimal VSN technique according to the supply battery's state of charge. Succeeding priorities are only forwarded, if VSN node's supply battery level is above the lowest threshold. For instance, raw data of the "flower" captured image is encoded as shown in Fig. 12, if an energy level (a) near maximum threshold, a VSN node adopts a scheme which will increase the probability of forwarding packets to $(75 \%)$ causing the lowest compression level is applied at 3.5556 with higher bit rate transmission (2.2500 bit per pixel). Such a policy will promote the reconstructed image quality to $39.0840 \mathrm{~dB}$ PSNR instead of energy savings. In opposition to what has beenstated, captured image packetized into lowest probability of (12.5\%) low frequency DCT coefficients, if an energy level (a) near minimum threshold 0 at highest compression level 21.3333 and lower bit rate transmission ( 0.3750 bit per pixel). This will promote energy savings instead of a higher resolution of the final reconstructed image at 23.0631 dB PSNR. This 
choice will depend on the application in which the VSN is involved and the predictable usage of the extracted images (Akyildiz et al., 2002).

In this study because the decision performed by a VSN node is completed independently of the available energy in the other VSN nodes, our suggested transmission system is competent as open loop which offerings excessive adaptation to all kinds of routing arrangement and its modeling and execution are very simple. (a)

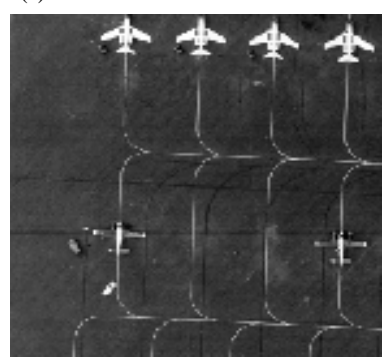

(d)

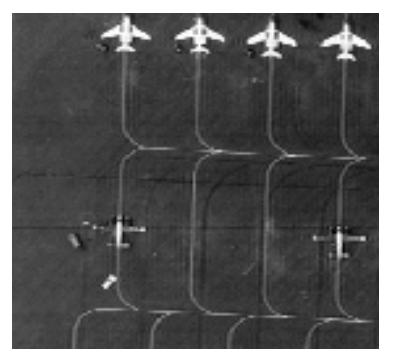

(b)

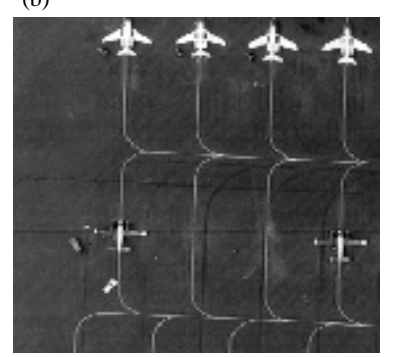

(e)

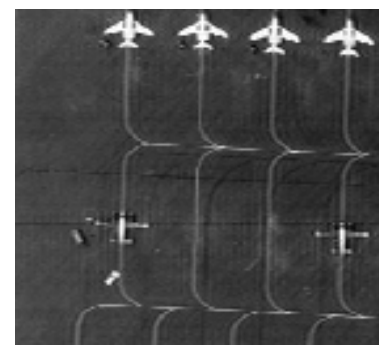

(g)

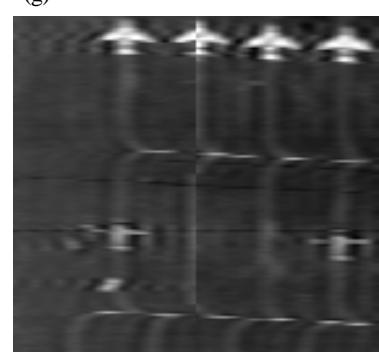

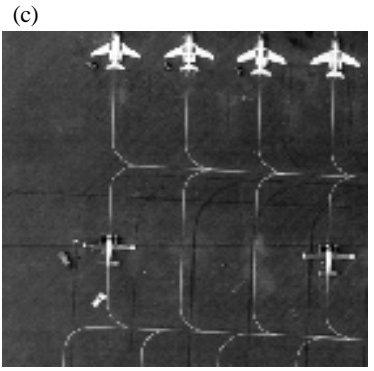

(f)

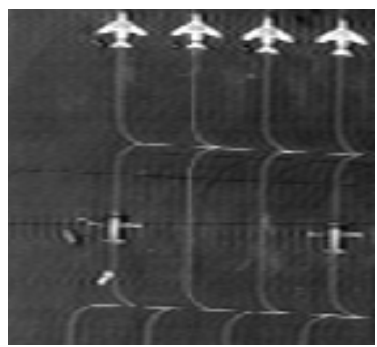

Fig. 8: a) Original image; b) Reconstructed image (75\%); c) Reconstructed image (50\%); d) Reconstructed image (40\%); e) Reconstructed image (30\%); f) Reconstructed image (25\%) and g) Reconstructed image (12.5\%)

(a)

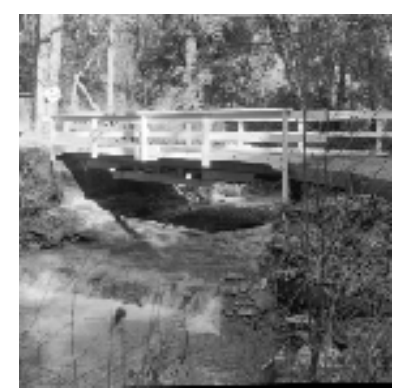

(b)

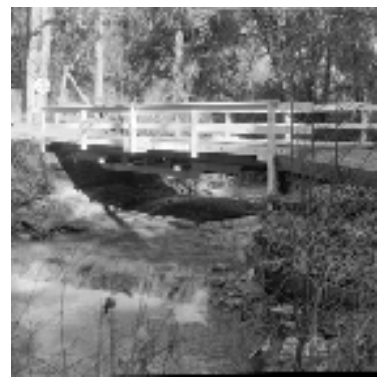

(c)

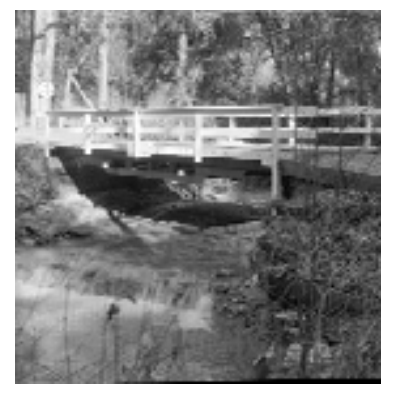

Fig. 9: Continue 
(d)

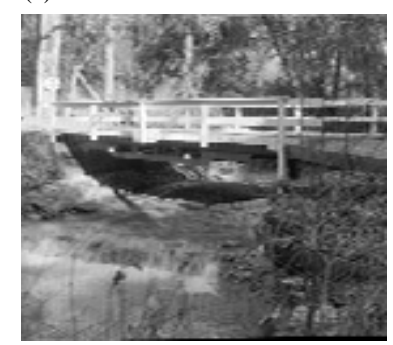

(e)

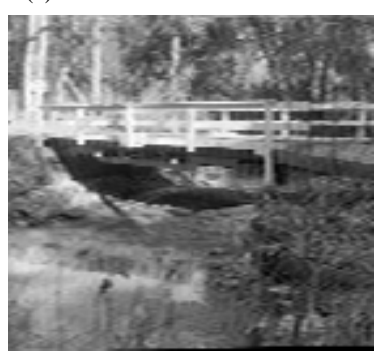

(g)
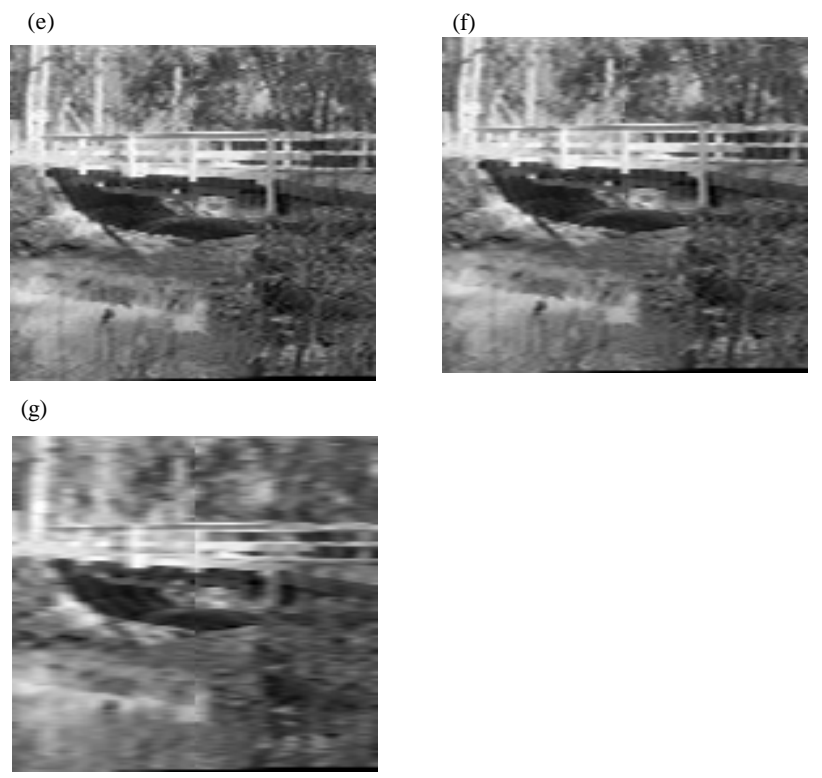

Fig. 9: a) Original image; b) Reconstructed image (75\%); c) Reconstructed image (50\%); d) Reconstructed image (40\%); e) Reconstructed image ( $30 \%)$; f) Reconstructed image ( $25 \%$ ) and g) Reconstructed image ( $12.5 \%)$
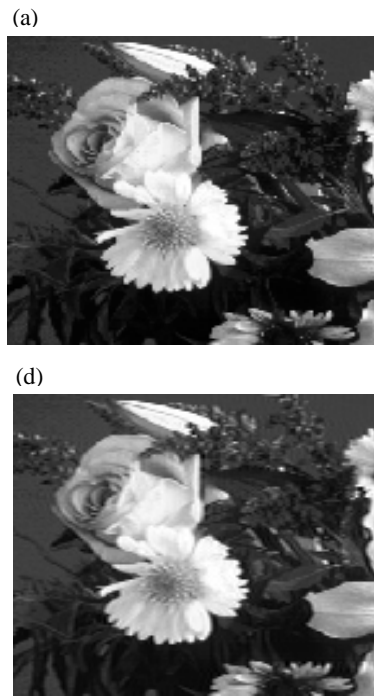

(b)

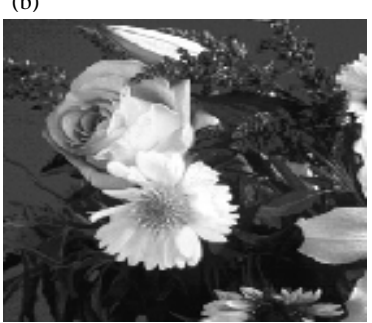

(e)

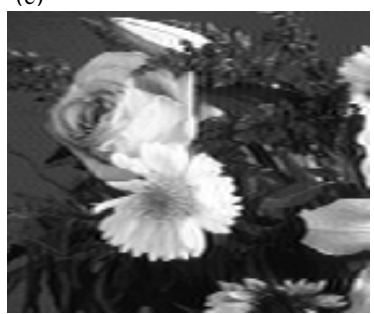

(g)

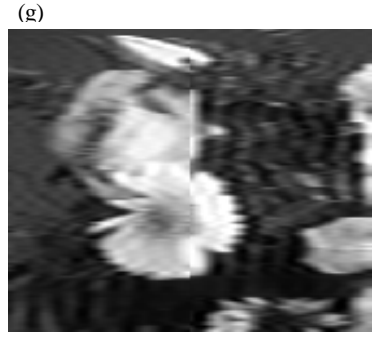

(c)

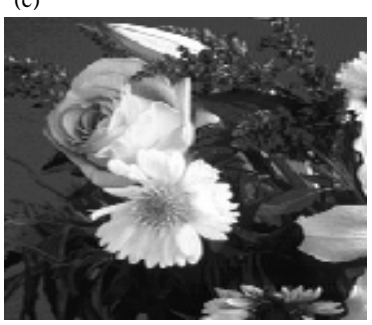

(f)

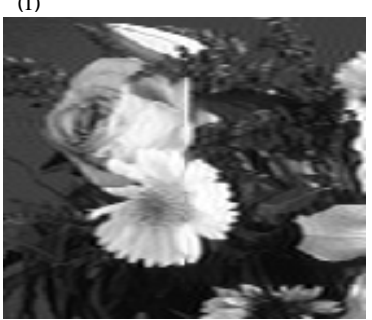

Fig. 10: a) Original image; b) Reconstructed image (75\%); c) Reconstructed image (50\%); d) Reconstructed image (40\%); e) Reconstructed image (30\%); f) Reconstructed image (25\%) and g) Reconstructed image (12.5\%) 


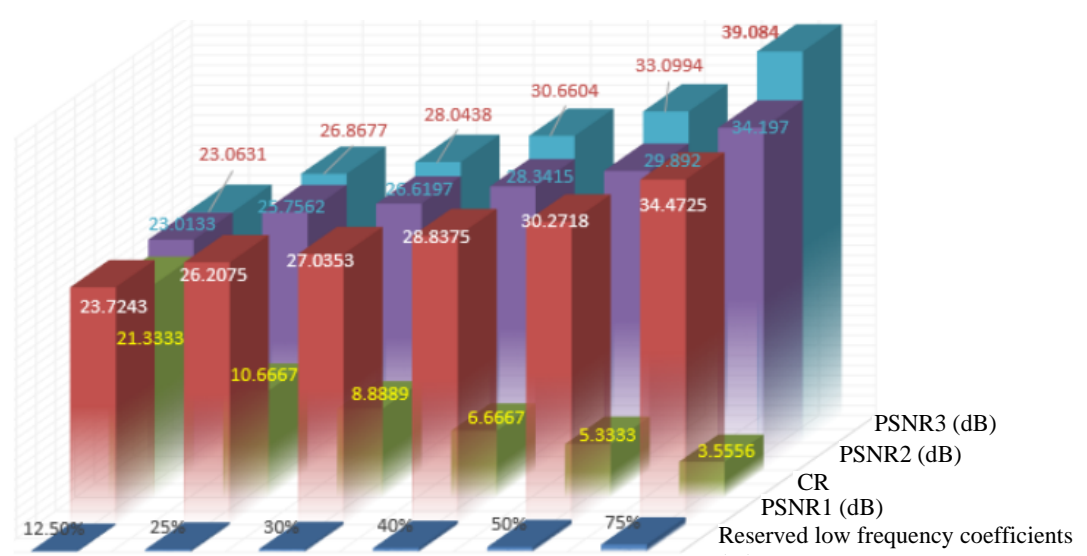

Fig. 11: A comparison between the three reconstructed images results

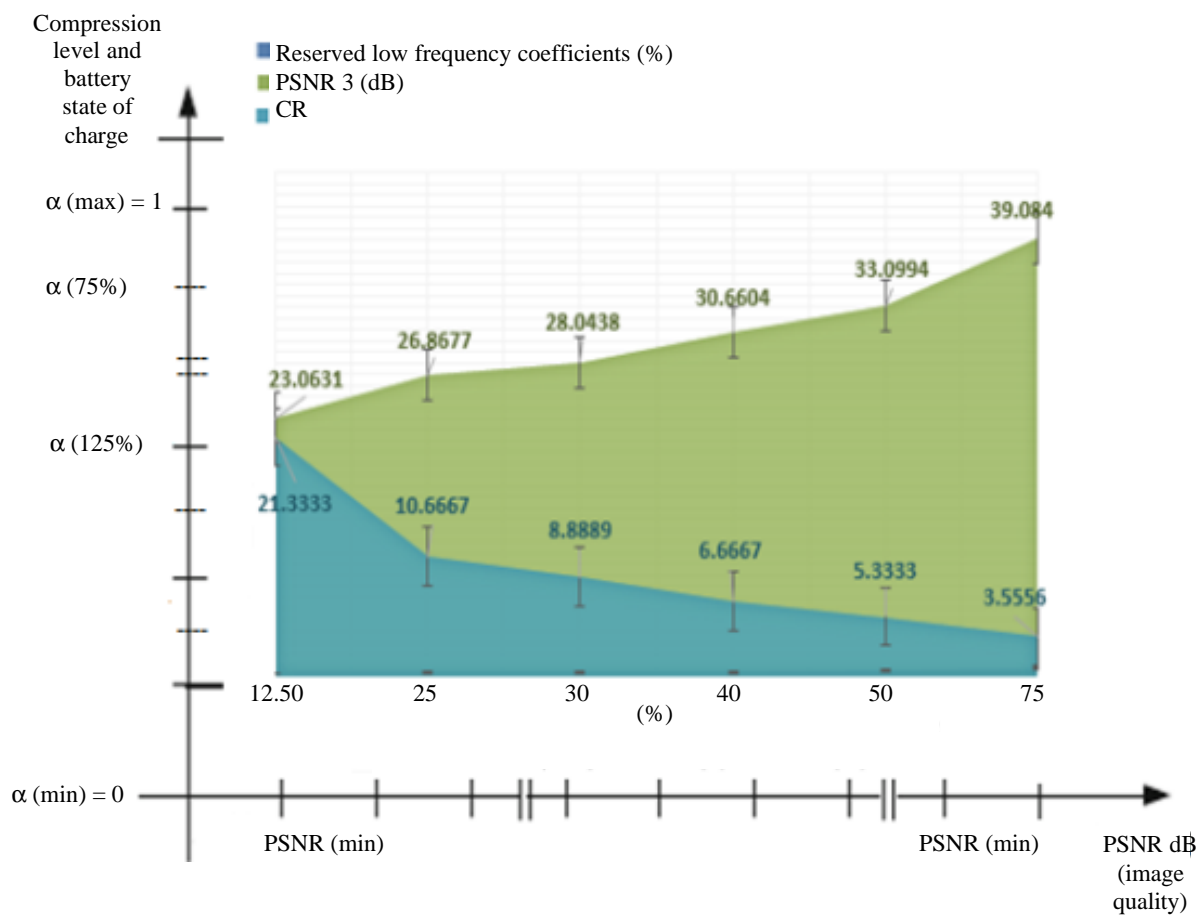

Fig. 12: VSN technique constraints policy based on PSNR and CR priorities process-based selection

\section{CONCLUSION}

This study has summarized a simple coding and effective energy depletion image compression system, i.e., discarding theory in VSNs. This approach reduces supply battery's energy consumption necessities meaningfully while retaining all the advantages achieved through modest adaptations to the JPEG Software. Furthermore, the discarding process of high frequency DCT coefficients provides a tradeoff between CR and image quality using DCT by segmenting each image line into vectors may result in blocking artifacts. These artifacts are perceptually annoying and become prominent in the reconstructed images at very low bit-rates. In this research, DCT based compression is investigated in the context of VSN at the sensor node. Nevertheless with less bits to transmit, this proposed technique will prolong the life of such networks and reduces supply battery's energy consumption.

\section{RECOMMENDATIONS}

In future research, closed loop plans will be explored to improve our proposal. A simulation will be delivered to provide more comprehensive and real results. Distributed 
and local compression algorithms will be considered to be combined in our proposal investigating their performances and their viability to be assimilated in a real wireless VSNs.

\section{ACKNOWLEDGEMENT}

The researchers wishes to express his sincerest gratitude to the Electrical Engineering Department, University of Tikrit for giving an opportunity to work on this study and for Engineering College contribution.

\section{REFERENCES}

Akyildiz, I.F., T. Melodia and K.R. Chowdhury, 2007. A survey on wireless multimedia sensor networks. Comput. Networks, 51: 921-960.

Akyildiz, I.F., W. Su, Y. Sankarasubramaniam and E. Cayirci, 2002. Wireless sensor networks: A survey. Comput. Netw., 38: 393-422.

Bracamonte, J. M. Ansorge and F. Pellandini, 1996. VLSI systems for image compression: A powerconsumption/image-resolution trade-off approach. Proceedings of the SPIE Conference on Digital Compression Technologies and Systems for Video Communications Vol. 2952, October 7-11, 1996, SPIE, Berlin, Germany, pp: 591-596.

Chew, L.W., L.M. Ang and K.P. Seng, 2008. Survey of image compression algorithms in wireless sensor networks. Proceedings of the 2008 International Symposium on Information Technology Vol. 4, Auguest 26-28, 2008, IEEE, Kuala Lumpur, Malaysia, ISBN:978-1-4244-2327-9, pp: 1-9.

Feig, E. and S. Winograd, 1992. Fast algorithms for the discrete cosine transform. IEEE. Trans. Signal Process., 40: 2174-2193.

Ferrigno, L., S. Marano, V. Paciello and A. Pietrosanto, 2005. Balancing computational and transmission power consumption in wireless image sensor networks. Proceedings of the IEEE Symposium on Virtual Environments, Human-Computer Interfaces and Measurement Systems, July 18-20, 2005, IEEE, Messina, Italy, pp: 1-6.

Hasan, K.K., U.K. Ngah and M.F.M. Salleh, 2014. Efficient hardware-based image compression schemes for wireless sensor networks: A survey. Wirel. Pers. Commun., 77: 1415-1436.

Jeong, H., J. Kim and W.K. Cho, 2004. Low-power multiplierless DCT architecture using image correlation. IEEE. Trans. Consum. Electron., 50: 262-267.

Liang, J. and T.D. Tran, 2001. Fast multiplierless approximations of the DCT with the lifting scheme. IEEE Trans. Signal Process., 49: 3032-3044.
Loeffler, C., A. Ligtenberg and G.S. Moschytz, 1989. Practical fast 1-D DCT algorithms with 11 multiplications. Proceedings of the International Conference on Acoustics, Speech and Signal Processing, May 23-26, 1989, Glasgow, Scotland, pp: 988-991.

Makkaoui, L., V. Lecuire and J.M. Moureaux, 2010. Fast zonal DCT-based image compression for wireless camera sensor networks. Proceedings of the 2nd International Conference on Image Processing Theory, Tools and Applications, July 7-10, 2010, IEEE, Paris, France, ISBN:978-1 -4244-7247-5, pp: 126129.

Mammeri, A., A. Khoumsi, D. Ziou and B. Hadjou, 2008b. Energy-efficient transmission scheme of JPEG images over visual sensor networks. Proceedings of the 2008 33rd IEEE Conference on Local Computer Networks (LCN), October 14-17, 2008, IEEE, Montreal, Que, Canada, ISBN:978-1-4244-2412-2, pp: 639-647.

Mammeri, A., A. Khoumsi, D. Ziou and B. Hadjou, 2008a. Modeling and adapting JPEG to the energy requirements of VSN. Proceedings of the 2008 Proceedings of 17 th International Conference on Computer Communications and Networks, Auguest 3-7, 2008, IEEE, Thomas, USA., pp: 1-6.

Nasri, M., A. Helali, H. Sghaier and H. Maaref, 2010. Adaptive image transfer for Wireless Sensor Networks (WSNs). Proceedings of the 5th International Conference on Design \& Technology of Integrated Systems in Nanoscale Era, March 23-25, 2010, IEEE, Hammamet, Tunisia, ISBN:978-1-42446338-1, pp: 1-7.

Pekhteryev, G., Z. Sahinoglu, P. Orlik and G. Bhatti, 2005. Image transmission over IEEE 802.15. 4 and Zigbee networks. Proceedings of the 2005 IEEE International Symposium on Circuits and Systems, May 23-26, 2005, IEEE, Kobe, Japan, pp: 3539-3542.

Srisooksai, T., K. Keamarungsi, P. Lamsrichan and K. Araki, 2012. Practical data compression in wireless sensor networks: A survey. J. Network. Comput. Applic., 35: 37-59.

Taylor, C.N. and S. Dey, 2001. Adaptive image compression for wireless multimedia communication. Proceedings of the ICC IEEE International Conference on Communications Conference Record (Cat. No.01CH37240 Vol. 6), June 11-14, 2001, IEEE, Helsinki, Finland, Finland, ISBN:0-7803-7097-1, pp: 1925-1929.

Wu, H. and A.A. Abouzeid, 2005. Energy efficient distributed image compression in resourceconstrained multihop wireless networks. Comput. Commun., 28: 1658-1668. 